\title{
Paeoniflorin inhibits proliferation and promotes apoptosis of multiple myeloma cells via its effects on microRNA-29b and matrix metalloproteinase-2
}

\author{
SHAOFENG WANG and WENHUA LIU \\ Department of Orthopedics, The Affiliated Hospital of Weifang Medical College, Weifang, Shandong 261031, P.R. China
}

Received January 25, 2015; Accepted October 29, 2015

DOI: $10.3892 / \mathrm{mmr} .2016 .5498$

\begin{abstract}
Multiple myeloma (MM) is a type of cancer characterized by the excessive proliferation of malignant plasma cells. In China, the incidence of MM has been increasing annually. Paeoniflorin exerts numerous functions, including coronary vessel expansion, and anti-inflammation and anticancer activities. The present study aimed to investigate the effects of paeoniflorin on the proliferation and apoptosis of SKO-007 MM cells, via its effects on the regulation of matrix metalloproteinase-2 (MMP-2) and microRNA (miR)-29b. In the present study, an MTT assay was used to analyze the proliferation of SKO-007 cells treated with paeoniflorin. Annexin V-fluorescein isothiocyanate/propidium iodide apoptosis and caspase- 3 activation assays were used to detect the levels of cellular apoptosis. The expression levels of MMP-2 and miR-29b were detected using gelatin zymography and quantitative-polymerase chain reaction, respectively. In addition, miR-29b and anti-miR-29b plasmids were transfected into SKO-007 cells, and the effects of paeoniflorin on cell proliferation and apoptosis were subsequently detected. The results of the present in vitro studies demonstrated that paeoniflorin was able to inhibit the proliferation of SKO-007 cells in a dose- and time-dependent manner. Furthermore, paeoniflorin effectively increased cell apoptosis, and augmented the activation of caspase- 3 and caspase- 9 in the SKO-007 cells. The expression levels of MMP-2 were suppressed following treatment of the SKO-007 cells with paeoniflorin. In addition, paeoniflorin was able to induce the expression of miR-29b. Notably, the results of the present study indicated that miR-29b expression may control the expression of MMP-2 in SKO-007 cells. In conclusion, the present study demonstrated that paeoniflorin was able to inhibit cell proliferation and promote apoptosis of MM cells
\end{abstract}

Correspondence to: Mr. Wenhua Liu, Department of Orthopedics, The Affiliated Hospital of Weifang Medical College, 2428 Yuhe Road, Weifang, Shandong 261031, P.R. China

E-mail: wenhualiu8036@163.com

Key words: paeoniflorin, multiple myeloma, microRNA-29b, matrix metalloproteinase-2 by suppressing the expression of MMP-2, via the upregulation of miR-29b.

\section{Introduction}

Multiple myeloma (MM), which is also known as plasma cell myeloma, is a type of cancer characterized by the excessive proliferation of malignant plasma cells, extensive osteolytic lesions, and/or osteoporosis (1). In recent years, among the hematological malignancies, the incidence of $\mathrm{MM}$ in the United States has overtaken acute leukemia, and is second only to non-Hodgkin's lymphoma (2). In addition, the incidence of MM in China has been increasing annually (3). MM is the most common primary tumor of the bone marrow in the USA $(4,5)$.

Matrix metalloproteinases (MMPs), which belong to the zinc-dependent endopeptidase family, exert proteolytic activity and are associated with bone remodeling, bone resorption, tumor invasion and metastasis $(6,7)$. Previous studies have also demonstrated that in myeloma cells, elevated levels of MMP-2 may be associated with disease progression, angiogenesis, and myeloma bone disease $(8,9)$. In addition, Zdzisińska et al (9) reported that suppression of MMP-2 activity was able to inhibit the proliferation of MM cells.

MicroRNAs (miRs) are small (19-25 nucleotides) non-coding RNA molecules that are involved in genetic regulation. miRs are involved in regulating various biological signaling pathways, and have several roles in the regulation of cell growth and development (10). miRs account for $1-2 \%$ of the known eukaryotic genome, and have a role in tumor biology, as either tumor suppressor genes or proto-oncogenes (11). Overexpression of miR-29b has previously been shown to reduce the protein expression levels of myeloid cell leukemia 1 (Mcl-1), thereby inhibiting the growth of MM cells. miR-29b has also been reported to inhibit the interleukin-6-induced upregulation of Mcl-1, thus suggesting that miR-29b may act as a tumor suppressor gene $(12,13)$.

Paeoniflorin exerts numerous functions, including sedation, spasmolysis, anti-inflammation, memory improvement, blood glucose-lowering effects, anti-emergency ulcer, coronary vessel expansion, anti-acute ovarian cancer and inhibition of cardiac ischemia and platelet aggregation (14). It has previously been demonstrated that paeoniflorin may effectively modulate the multidrug resistance of the SGC7901 human gastric cancer cell line, via inhibition of nuclear factor $-\kappa \mathrm{B}$ activation (15). 
Furthermore, paeoniflorin has been reported to significantly induce the apoptosis of HeLa human cervical cancer cells, via the downregulation of B-cell lymphoma-2 (Bcl-2) and the upregulation of caspase-3 (16). However, whether paeoniflorin affects the proliferation and apoptosis of SKO-007 MM cells via suppression of MMP-2 expression and upregulation of miR-29b remains unknown. The present study aimed to investigate the effects and underlying molecular mechanisms of paeoniflorin on MM cell proliferation and apoptosis.

\section{Materials and methods}

Reagents and chemicals. The chemical structure of paeoniflorin (Sigma-Aldrich, St. Louis, MO, USA; purity $>98 \%$ ) is presented in Fig. 1. Paeoniflorin was dissolved in physiological saline, according to the manufacturer's protocol (17). RPMI-1640 mediumand fetal bovine serum(FBS) were purchased from Gibco (ThermoFisherScientific,Inc.,Waltham,MA,USA).Caspase-3/9 Activity Assay kits and Annexin V-Fluorescein Isothiocyanate (FITC)/Propidium Iodide (PI) Apoptosis Detection kit were purchased from Nanjing KeyGen Biotech Co., Ltd. (Nanjing, China). TRIzol reagent and quantitative-polymerase chain reaction (qPCR) assays were purchased from Invitrogen (Thermo Fisher Scientific, Inc.).

Cell culture. The SKO-007 human MM cell line (Type Culture Collection of the Chinese Academy of Sciences, Shanghai, China) was cultured in RPMI-1640 medium supplemented with $10 \%$ FBS, 100 units $/ \mathrm{ml}$ penicillin and $100 \mathrm{mg} / \mathrm{ml}$ streptomycin (Invitrogen; Thermo Fisher Scientific, Inc.) at $37^{\circ} \mathrm{C}$ in a humidified atmosphere containing $5 \% \mathrm{CO}_{2}$. The culture media were changed every 2-3 days.

Cell viability assay. SKO-007 cells were seeded at a density of $1 \times 10^{4} /$ well into 96 -well plates, and were treated with paeoniflorin $(0,5,10$ and $20 \mu \mathrm{M})$ for $48 \mathrm{~h}$. Next, GM60001 $(5 \mu \mathrm{M})$, was used to suppress the expression of MMP-2 and added to SKO-007 cells for $48 \mathrm{~h}$. Subsequently, cell viability was measured using the 3-(4,5-dimethylthiazol-2-yl)-2,5-diphenyltetrazolium bromide (MTT; Beyotime Institute of Biotechnology, Jiangsu, China) assay. Briefly, $15 \mu \mathrm{l}$ MTT was added to each well and incubated for $4 \mathrm{~h}$ at $37^{\circ} \mathrm{C}$ in a humidified atmosphere containing $5 \% \mathrm{CO}_{2}$. Subsequently, $150 \mu 1$ dimethyl sulfoxide was added to each well, and the plates were agitated for $20 \mathrm{~min}$. The absorbance of each well was measured at $\lambda=570 \mathrm{~nm}$ using a Varioskan Flash Multimode Reader (Thermo Fisher Scientific, Inc.).

Lactate dehydrogenase (LDH) assay. SKO-007 cells were seeded at a density of $1 \times 10^{4} /$ well into $96-$ well plates, and were treated with paeoniflorin $(0,5,10$ and $20 \mu \mathrm{M})$ for $0,24,48$ and $72 \mathrm{~h}$. Subsequently, the cytotoxicity of paeoniflorin to SKO-007 cells was measured using an LDH assay (Beyotime Institute of Biotechnology). Briefly, $100 \mu \mathrm{LDH}$ was added to each well and incubated for $30 \mathrm{~min}$ at room temperature. The absorbance was then measured at $490 \mathrm{~nm}$ using a multi-well spectrophotometer (868; BioTek Instruments, Inc., Winooski, VT, USA).

Annexin V-FITC/PI apoptosis assay. SKO-007 cells were seeded at a density of $1 \times 10^{6} /$ well into 6 -well plates, and were treated with paeoniflorin $(0,5,10$ and $20 \mu \mathrm{M})$ for

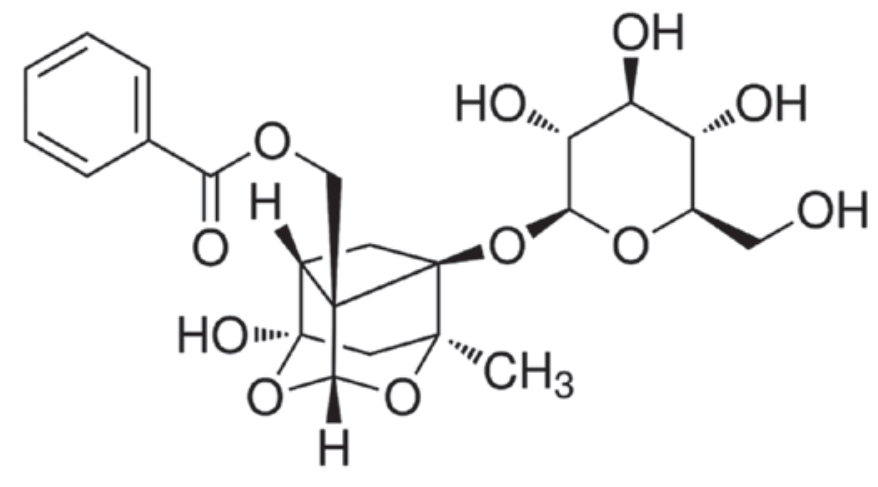

Figure 1. Chemical structure of paeoniflorin.

$48 \mathrm{~h}$. The number of apoptotic cells was measured using an Annexin V-FITC/PI Apoptosis Detection kit. Briefly, SKO-007 cells were washed twice with phosphate-buffered saline and resuspended in $1 \mathrm{X}$ binding buffer. Annexin V-FITC (10 $\mu \mathrm{l})$ was then added and incubated for $10 \mathrm{~min}$ at room temperature in the dark. Subsequently, PI (5 $\mu 1)$ was added and the cells were incubated for a further $10 \mathrm{~min}$ at room temperature in the dark. A flow cytometer (FACScalibur; BD Biosciences, San Jose, CA, USA) and CellQuest ${ }^{\mathrm{TM}}$ Pro software (BD Biosciences) were used to analyze the rate of apoptosis.

Caspase-3 and caspase-9 activation assay. SKO-007 cells were seeded at a density of $1 \times 10^{4} /$ well into 96 -well plates, and were treated with paeoniflorin $(0,5,10$ and $20 \mu \mathrm{M})$ for 48 h. Caspase- 3 and caspase- 9 activities were measured using Caspase-3/9 Activity Assay kits, according to the manufacturer's protocol. Briefly, $10 \mu \mathrm{l}$ protein cell lysate (containing $50 \mathrm{mM}$ Tris- $\mathrm{HCl} \mathrm{pH} 8.0,5 \mathrm{mM}$ EDTA, $150 \mathrm{mM} \mathrm{NaCl}, 1 \%$ Triton-X 100; Beyotime Institute of Biotechnology) per sample was added to $100 \mu \mathrm{l}$ reaction buffer containing $10 \mu \mathrm{l}$ substrate (Ac-DEVD-pNA for caspase-3, and Ac-LEHD-pNA for caspase-9) and incubated at $37^{\circ} \mathrm{C}$ for $4-6 \mathrm{~h}$. Caspase-3 and caspase- 9 activities were measured at an absorbance of $405 \mathrm{~nm}$.

Gelatin zymography. SKO-007 cells were seeded at a density of $1 \times 10^{6} /$ well into 6 -well plates, and were treated with paeoniflorin $(0,5,10$ and $20 \mu \mathrm{M})$ for $48 \mathrm{~h}$. The expression levels of MMP-2 in the SKO-007 cells were determined using zymographic analysis. Briefly, every sample was subjected to $10 \%$ sodium dodecyl sulfate (SDS)-polyacrylamide gel electrophoresis containing $0.1 \%$ gelatin. Following electrophoresis, the gels were washed with $50 \mathrm{mM}$ Tris- $\mathrm{HCl}$ (pH 7.6) three times for $30 \mathrm{~min}$ at room temperature, in order to remove the SDS. The gels were then incubated in a reaction buffer (Beyotime Institute of Biotechnology) for $12 \mathrm{~h}$ at $37^{\circ} \mathrm{C}$, after which, the gel was stained with $0.1 \%$ Coomassie Brilliant Blue G250 (Aladdin Industrial Corporation, Shanghai, China) for $1 \mathrm{~h}$ and destained in $10 \%$ acetic acid and $10 \%$ methanol.. THe resulting gels were analyzed using a film processor (CP 1000; Agfa-Gevaert N.V, Mortsel, Belgium).

Reverse transcription-qPCR analysis of miR-29b expression. SKO-007 cells were seeded at a density of $1 \times 10^{6} /$ well into 6 -well plates, and were treated with paeoniflorin $(0,5,10$ and 
$20 \mu \mathrm{M}$ ) for $48 \mathrm{~h}$. Total RNA was extracted from the cells using TRIzol reagent, and miRs were specifically amplified for quantification using individual miRNA TaqMan Real-Time qPCR analysis (Invitrogen; Thermo Fisher Scientific, Inc.). The primer sequences were as follows: miR-29b, forward 5'-GGGGGTACCCTTCAGGAAGCTGGTTTC-3', reverse 5'-GGGGATATCTACATGTGAGGCAGGTTCTCAC-3'; and U6, forward 5'-CGCTTCGGCAGCACATATACTA-3', and reverse 5'-CGCTTCACGAATTTGCGTGTCA-3'. The PCR conditions were $95^{\circ} \mathrm{C}$ for $60 \mathrm{sec}, 95^{\circ} \mathrm{C}$ for $30 \mathrm{sec}, 60^{\circ} \mathrm{C}$ for $45 \mathrm{sec}, 72^{\circ} \mathrm{C}$ for $30 \mathrm{~min}, 55^{\circ} \mathrm{C}$ for $30 \mathrm{sec}$, for 35 cycles. The results were normalized to $\beta$-actin. $\Delta \mathrm{Cq}$ was calculated by subtracting the $\mathrm{Ct}$ of U6. The $\Delta \Delta \mathrm{Cq}$ was then calculated by subtracting the $\Delta \mathrm{Cq}$ of the negative control from the $\Delta \mathrm{Cq}$ of the samples using the $2^{-\Delta \Delta \mathrm{Cq}}$ method (18).

Transfection of miR-29b and anti-miR-29b plasmids. miR-29b and anti-miR-29b plasmids and their negative controls were designed and synthesized by Sangon Biotech Co., Ltd. (Shanghai, China). Sequences for miR-29b plasmid 5'-GGG GGTACCCTTCAGGAAGCTGGTTTC-3' and 5'-GGGGAT ATCTACATGTGAGGCAGGTTCTCAC-3' and anti-miR-29b plasmid 5'-ACTGATTTCAAATGGTGCT-3' and 5'-GTGTAA CACGTCTATACGCCCA-3' Once the SKO-007 cells, which were seeded into 6 -well plates $\left(1 \times 10^{5}\right.$ cells/well), had reached $50-60 \%$ confluence, the plasmids were transfected into the cells using Lipofectamine 2000 (Invitrogen; Thermo Fisher Scientific, Inc.), according to the manufacturer's protocol.

Statistical analysis. All experiments were repeated three times, in order to ensure reproducibility. The data are presented as the mean \pm standard deviation, and were analyzed using SPSS 18 (SPSS Inc., Chicago, IL. USA). Comparisons between mean values were tested using Student's unpaired t-test. $\mathrm{P}<0.05$ was considered to indicate a statistically significant difference.

\section{Results}

Paeoniflorin inhibits the proliferation of SKO-007 cells. The effects of paeoniflorin were examined on the proliferation of the SKO-007 human MM cell line. Treatment with paeoniflorin inhibited the proliferation of SKO-007 cell in a dose- and time-dependent manner (Fig. 2). When the cells were treated with $5 \mu \mathrm{M}$ paeoniflorin for $72 \mathrm{~h}, 10 \mu \mathrm{M}$ paeoniflorin for 48 or $72 \mathrm{~h}$, or $20 \mu \mathrm{M}$ paeoniflorin for 24,48 or $72 \mathrm{~h}$, cell proliferation was significantly reduced, as compared with in the untreated cells (Fig. 2).

Paeoniflorin exerts cell cytotoxic effects on SKO-007 cells. To determine whether paeoniflorin was cytotoxic to SKO-007 cells, an LDH assay was conducted following treatment with paeoniflorin $(0,5,10$ and $20 \mu \mathrm{M})$ for $48 \mathrm{~h}$. As indicated in Fig. 3, paeoniflorin exerted cell cytotoxic effects on SKO-007 cells in a dose-dependent manner. The cell cytotoxic effects of paeoniflorin on SKO-007 cells markedly increased following treatment with 10 or $20 \mu \mathrm{M}$ paeoniflorin for $48 \mathrm{~h}$ (Fig. 3).

Paeoniflorin induces apoptosis of SKO-007 cells. To investigate whether paeoniflorin was able to promote SKO-007

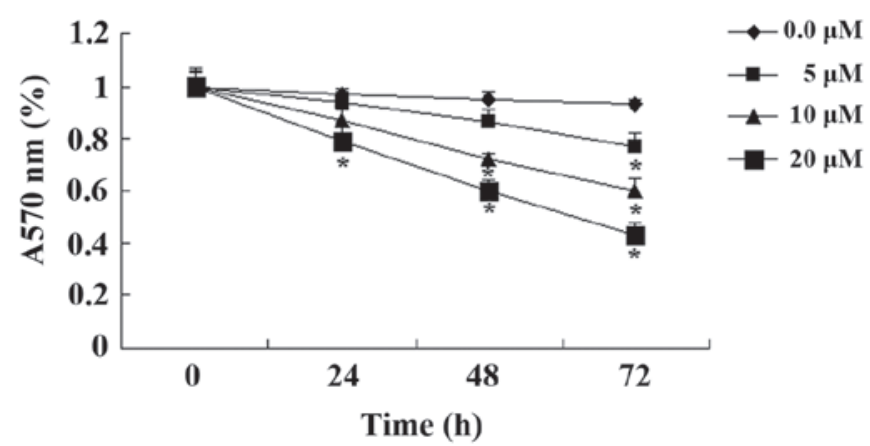

Figure 2. Paeoniflorin inhibited the proliferation of SKO-007 human multiple myeloma cells. Data are presented as the mean \pm standard deviation. ${ }^{*} \mathrm{P}<0.01$, compared with the $0 \mu \mathrm{M}$ paeoniflorin treatment group.

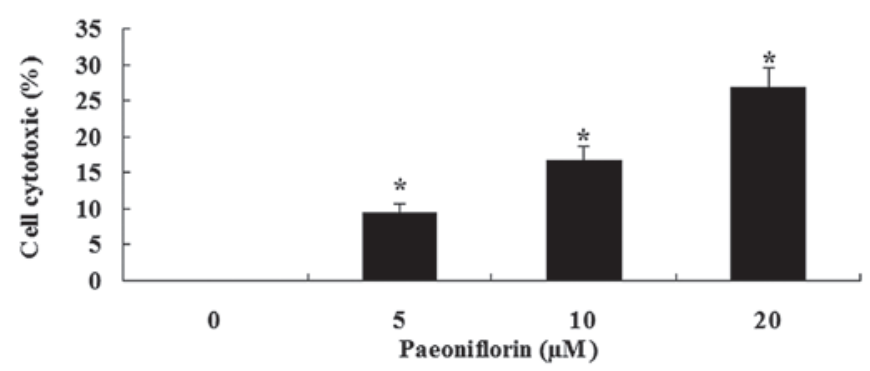

Figure 3. Paeoniflorin exerted cell cytotoxic effects on SKO-007 human multiple myeloma cells. Data are presented as the mean \pm standard deviation. ${ }^{*} \mathrm{P}<0.05$.

cell apoptosis, the number of apoptotic cells was determined using an Annexin V-FITC/PI apoptosis assay. Treatment with paeoniflorin $(0,5,10$ and $20 \mu \mathrm{M})$ for $48 \mathrm{~h}$ induced a concentration-dependent increase in the rate of SKO-007 cell apoptosis (Fig. 4A and B). Following treatment with 10 or $20 \mu \mathrm{M}$ paeoniflorin, the number of SKO-007 apoptotic cells was significantly increased (Fig. 4A and B).

Paeoniflorin induces caspase-3 and caspase-9 activities in SKO-007 cells. To further investigate the effects of paeoniflorin on the caspase activity of SKO-007 cells, caspase-3 and caspase-9 activities were measured using Caspase-3/9 Activity Assay kits. Notably, treatment with paeoniflorin (10 and $20 \mu \mathrm{M}$ ) for $48 \mathrm{~h}$ induced caspase-3 and caspase-9 activities in SKO-007 cells (Fig. 5A and B).

Paeoniflorin inhibits MMP-2 protein expression levels in SKO-007 cells. The present study aimed to determine whether paeoniflorin was able to inhibit the expression levels of MMP-2 in SKO-007 cells. Treatment with paeoniflorin (10 and $20 \mu \mathrm{M})$ for $48 \mathrm{~h}$ markedly inhibited the protein expression levels of MMP-2 in the SKO-007 cells (Fig. 6A and B).

Suppression of $M M P-2$ reduces the proliferation of paeoniflorin-treated SKO-007 cells. To determine the potential regulatory mechanisms underlying the effects of paeoniflorin $(10 \mu \mathrm{M})$ on the proliferation of SKO-007 cells after $48 \mathrm{~h}$, the cells were treated with an MMP-2 inhibitor, GM6001 (5 $\mu \mathrm{M}$; Chemicon International, Temecula, CA, 
A Paeoniflorin $(\mu \mathrm{M})$
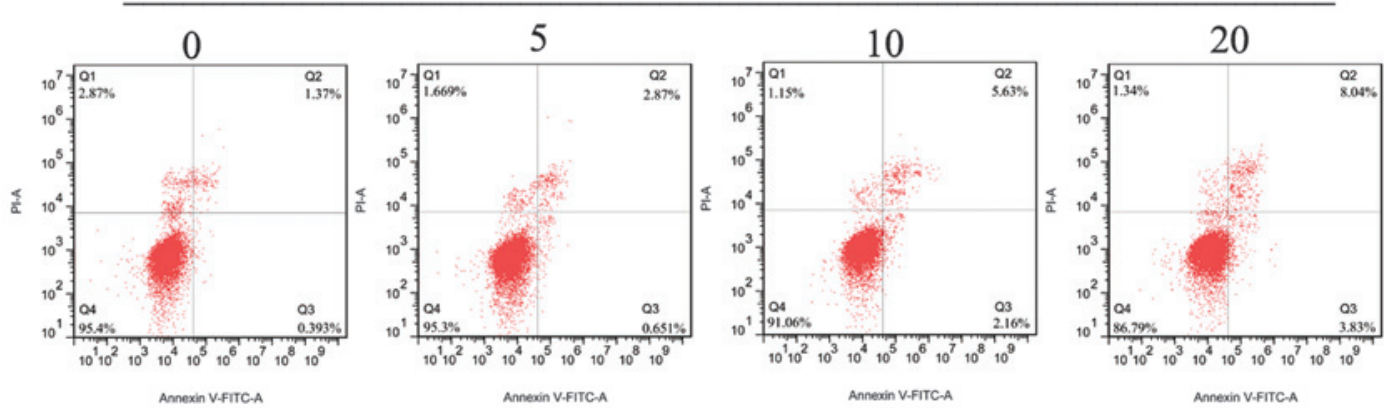

B
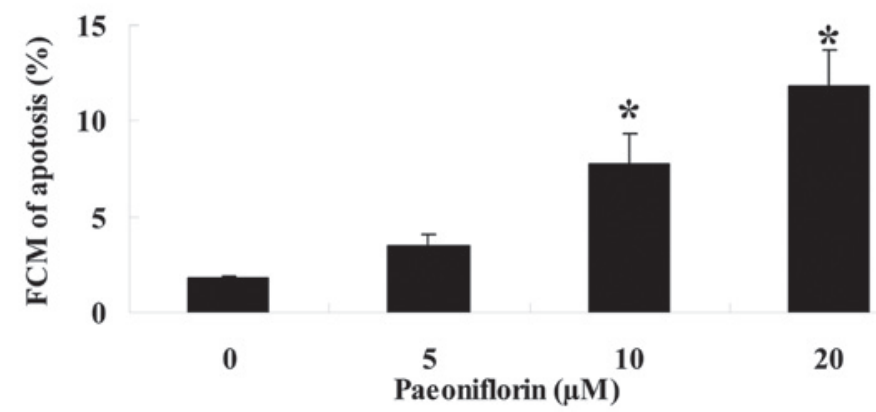

20

Figure 4. Paeoniflorin promoted cellular apoptosis of SKO-007 human multiple myeloma cells. (A) Paeoniflorin induced apoptosis of SKO-007 cells. (B) Statistical analysis of the rate of cellular apoptosis. Data are presented as the mean \pm standard deviation. ${ }^{*} \mathrm{P}<0.01$, compared with the $0 \mu \mathrm{M}$ paeoniflorin treatment group. FCM, flow cytometry.

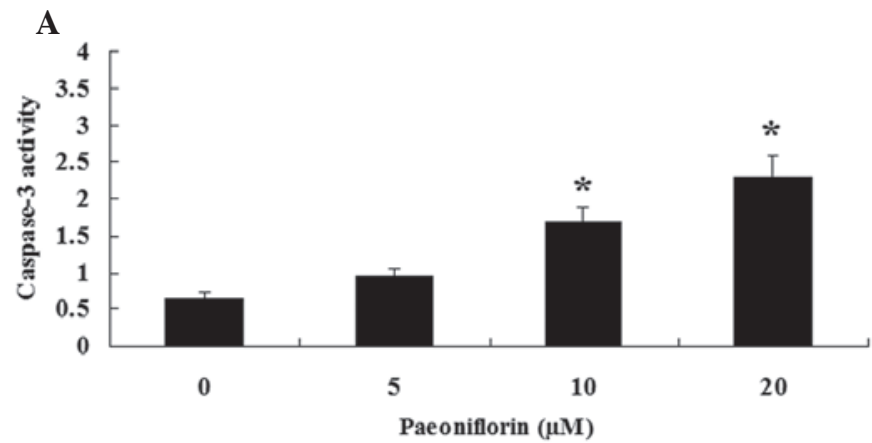

B

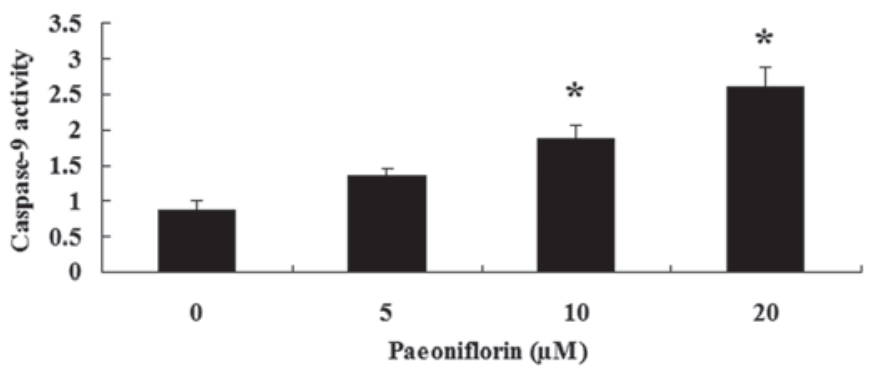

Figure 5. Paeoniflorin induced (A) caspase-3 and (B) caspase-9 activity in SKO-007 human multiple myeloma cells. Data are presented as the mean \pm standard deviation. ${ }^{*} \mathrm{P}<0.01$, compared with the $0 \mu \mathrm{M}$ paeoniflorin treatment group.

USA). Treatment of the cells with the MMP-2 inhibitor suppressed the protein expression levels of MMP-2, and further reduced the proliferation of paeoniflorin-treated cells (Fig. 7A-C).

Paeoniflorin upregulates miR-29b expression in SKO-007 cells. The present study aimed to investigate whether paeoniflorin was able to activate miR-29b expression in SKO-007 cells. Following treatment with paeoniflorin $(10$ and $20 \mu \mathrm{M})$ for $48 \mathrm{~h}$, the expression levels of miR-29b were significantly increased in the SKO-007 cells (Fig. 8).

Overexpression of miR-29b inhibits $M M P-2$ expression in SKO-007 cells. SKO-007 cells were transfected with miR-29b, in order to determine whether overexpression of miR-29b was able to inhibit the expression of MMP-2. Transfection of the cells with miR-29b increased the expression levels of miR-29b and suppressed the expression of MMP-2 in SKO-007 cells (Fig. 9A and B).

Anti-miR-29b attenuates the effects of paeoniflorin. To determine whether a correlation exists between the effects of paeoniflorin and miR-29b expression, the effects of paeoniflorin on SKO-007 cells transfected with anti-miR-29b were investigated. Transfection with an anti-miR-29b antibody significantly reduced the expression levels of miR-29b in SKO-007 cells (Fig. 10A). Notably, transfection with anti-miR-29b significantly attenuated the effects of paeoniflorin on cell proliferation (Fig. 10B) and apoptosis (Fig. 10C) in SKO-007 cells. Furthermore, transfection with anti-miR-29b was able to upregulate the expression levels of MMP-2 in SKO-007 cells (Fig. 10D). 
A

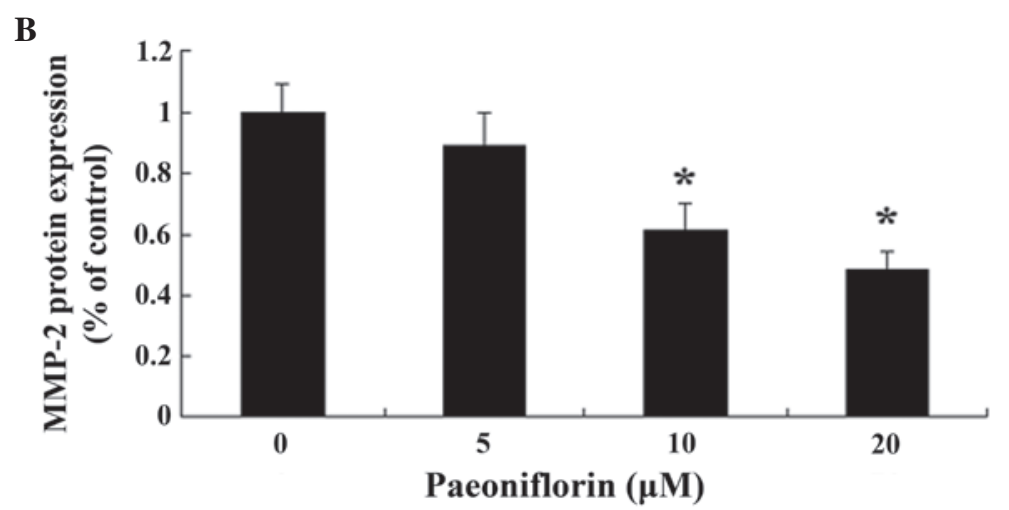

Figure 6. Paeoniflorin inhibited the protein expression levels of MMP-2 in the SKO-007 human multiple myeloma cells. The effects of paeoniflorin on (A) MMP-2 expression. (B) Statistical analysis of MMP-2 protein expression levels. Data are presented as the mean \pm standard deviation. " $\mathrm{P}<0.01$, compared with the $0 \mu \mathrm{M}$ paeoniflorin treatment group. MMP-2, matrix metalloproteinase-2.

A

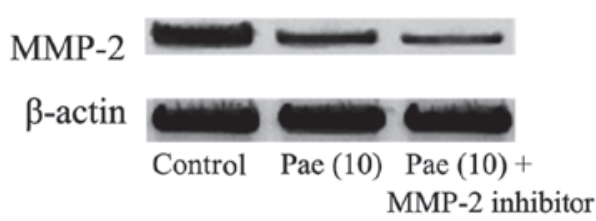

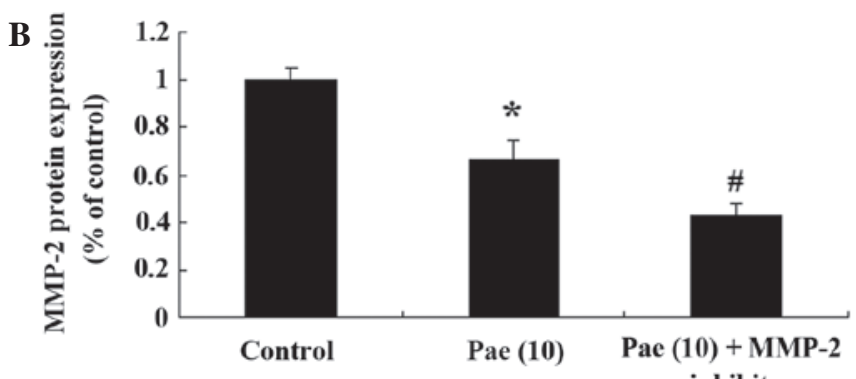

inhibitor

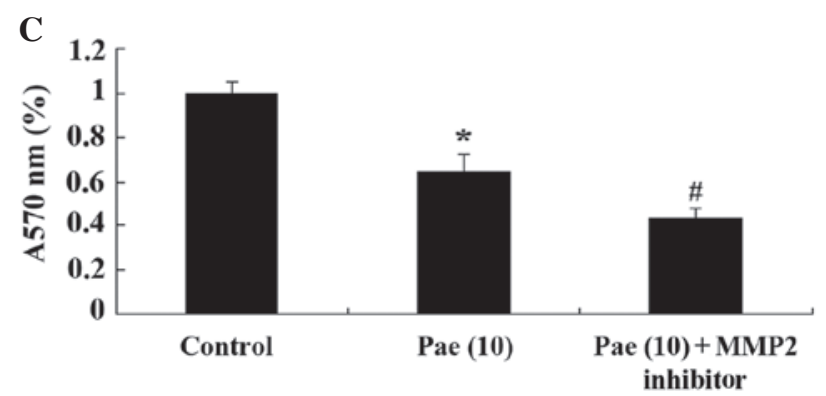

Figure 7. MMP-2 suppression further reduced the proliferation of paeoniflorin-treated SKO-007 human multiple myeloma cells. Treatment with the MMP-2 inhibitor (A) inhibited MMP-2 expression, (B) statistical analysis of MMP-2 protein expression; and (C) further reduced the proliferation of paeoniflorin-treated cells. Data are presented as the mean \pm standard deviation. " $\mathrm{P}<0.01$, compared with the $0 \mu \mathrm{M}$ paeoniflorin treatment group; ${ }^{*} \mathrm{P}<0.01$, compared with the $10 \mu \mathrm{M}$ paeoniflorin treatment group. MMP-2, matrix metalloproteinase-2; Pae, paeoniflorin.

\section{Discussion}

MM is a debilitating and incurable malignant disease, which originates in $\mathrm{B}$ cells. MM is characterized by the malignant proliferation and abnormal accumulation of bone marrow clonal plasma cells, and is associated with increased monoclonal immunoglobulin levels $(19,20)$. In the present study, treatment with paeoniflorin was able to inhibit the proliferation of SKO-007 cells in a dose- and time-dependent manner. Paeoniflorin is a promising agent in the treatment of liver cancer via the downregulation of prostaglandin E2 receptor 2 expression and the increased activation of caspase-3 (21). In addition, in the present study paeoniflorin significantly enhanced cell cytotoxicity, increased apoptosis, and accelerated caspase-3 activation in SKO-007 cells. Zhang and Zhang (17) reported that paeoniflorin was able to significantly induce the apoptosis of HeLa cells, via the downregulation of Bcl-2 and the

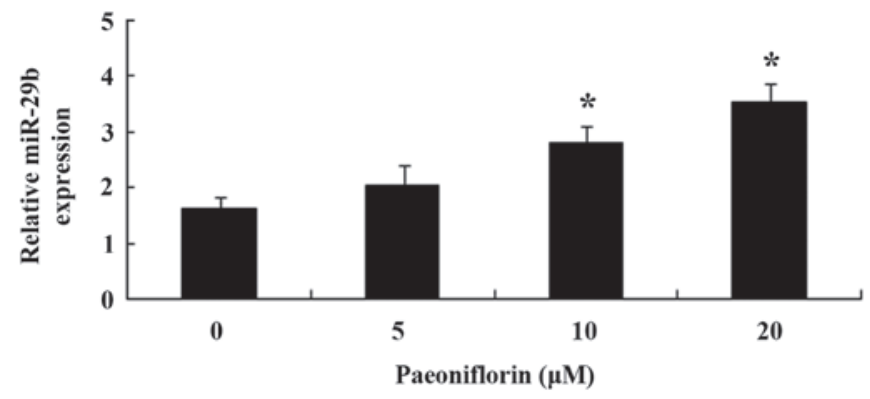

Figure 8. Paeoniflorin upregulated miR-29b expression in SKO-007 human multiple myeloma cells. Data are presented as the mean \pm standard deviation. ${ }^{*} \mathrm{P}<0.01$, vs. the $0 \mu \mathrm{M}$ paeoniflorin treatment group. miR-29b, microRNA-29b.

upregulation of caspase-3 (22). Hung et al (23) demonstrated that the antiproliferative activity of paeoniflorin promoted the apoptosis of A549 human non-small cell lung cancer cells. 
A

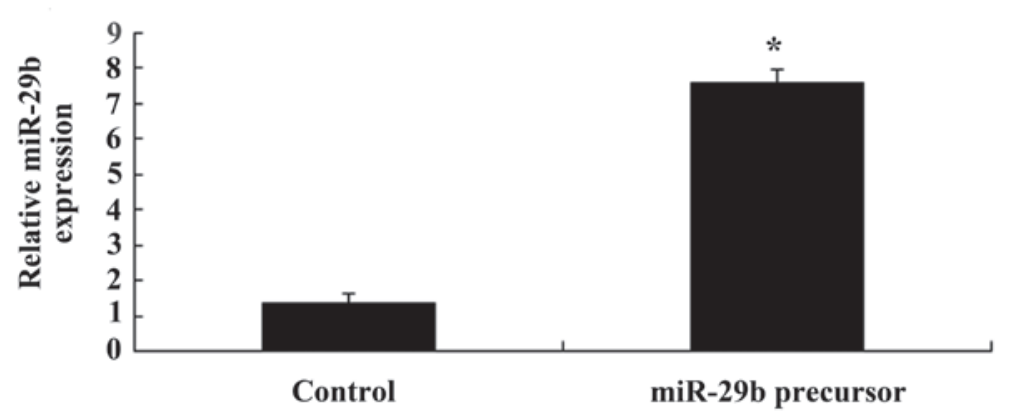

B

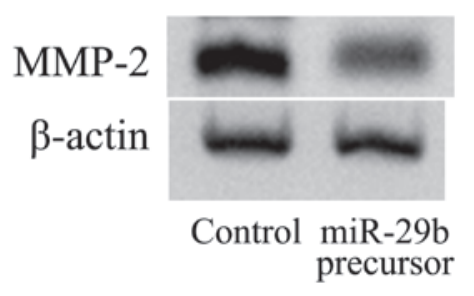

Figure 9. Overexpression of miR-29b (A) increased miR-29b expression and (B) inhibited MMP-2 protein expression in SKO-007 human multiple myeloma cells. Data are presented as the mean \pm standard deviation. ${ }^{*} \mathrm{P}<0.01$, compared with the $0 \mu \mathrm{M}$ paeoniflorin treatment group. MMP-2, matrix metalloproteinase-2; miR-29b, microRNA-29b.

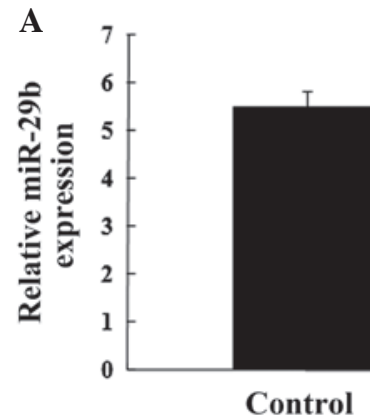

A
C

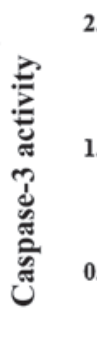

Paeoniflorin

Negative control -

Anti-miR-29b

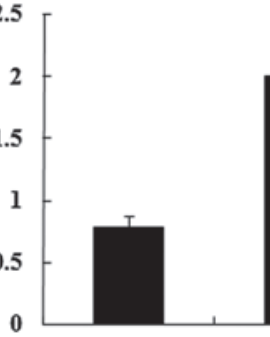

-
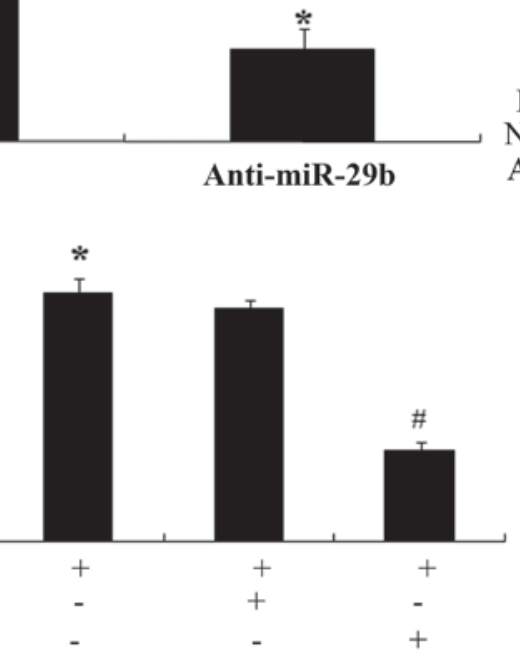

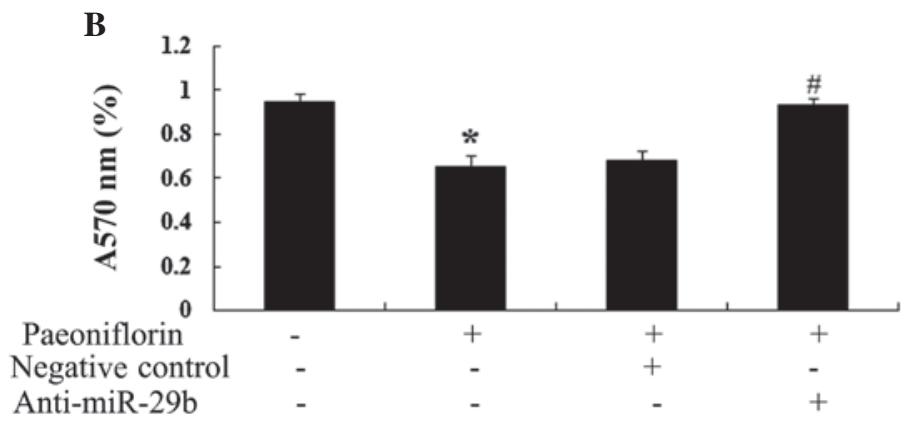

D

\section{MMP-2}

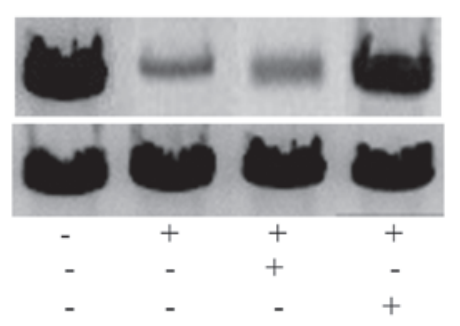

Figure 10. (A) Anti-miR-29b reversed the effects of paeoniflorin on the expression levels of miR-29b in SKO-007 human multiple myeloma cells. Following treatment with paeoniflorin $(10 \mu \mathrm{M})$ for $48 \mathrm{~h}$, anti-miR-29b significantly (B) promoted the cell proliferation and (C) inhibited the cell apoptosis of SKO-007 cells. (D) Anti-miR-29b significantly increased MMP-2 activity in SKO-007 cells following treatment with paeoniflorin (10 $\mu \mathrm{M})$ for $48 \mathrm{~h}$. Data are presented as the mean \pm standard deviation. ${ }^{*} \mathrm{P}<0.01$, compared with the $0 \mu \mathrm{M}$ paeoniflorin treatment group; ${ }^{*} \mathrm{P}<0.01$, compared with the paeoniflorin-treated group transfected with negative control. MMP-2, matrix metalloproteinase-2; miR-29b, microRNA-29b.

However, further research regarding the underlying molecular mechanisms of paeoniflorin on MM cell proliferation and apoptosis is required.

MMP-2 is usually located in the cytoplasm of tumor cells, and is often detected in the endothelial cells of the vascular basement membrane, thus suggesting that during the invasion of astrocytoma, MMP-2 and vascular endothelial growth factor have a synergistic role in regulating the angiogenesis of tumor vessels in MM $(24,25)$. The structure of newly formed tumor vasculature is incomplete, and easily leaks, thus affecting the integrity of the blood-brain barrier (BBB); plasma and other macromolecules may penetrate into the cells through the damaged BBB and gather around the tumor, forming extensive peritumoral edema (26). Furthermore, MMP-2 may directly degrade the extracellular matrix of the basement membrane of blood vessels, loosening the structure, which is conducive to the invasion of tumor cells along the basement membrane (27). The results of the present study revealed that paeoniflorin was able to inhibit the expression levels of MMP-2 in SKO-007 cells. In addition, downregulation of MMP-2 further reduced the proliferation of paeoniflorin-treated SKO-007 cells. A previous study demonstrated that paeoniflorin inhibits the MMP-2 activity of splenocytes from picryl chloride-induced ear contact sensitivity mice (28).

In MM, tumor cells proliferate in the surrounding bone marrow, promoting the activities and inhibiting the function of osteoclasts. In experiments on peripheral blood samples, the expression of miR-29b is decreased during osteoclast differentiation, and is therefore considered a negative regulator 
of human osteoclast differentiation and activity (29). Furthermore, miR-29b inhibits the activity of tartrate-resistant acid phosphatase on osteoclasts and affects osteoclast induced bone resorption through reducing MMP-9 expression $(30,31)$. The present study demonstrated that paeoniflorin significantly increased the expression levels of miR-29b in SKO-007 cells. Simultaneously, overexpression of miR-29b was capable of inhibiting the expression of MMP-2 in SKO-007 cells. These results indicated that the expression of miR-29b may regulate and control MMP-2 expression in SKO-007 cells; therefore, the miR-29b/MMP-2 signaling pathway may be considered a novel pharmaceutical target for the treatment of MM using paeoniflorin.

In conclusion, the key observation of the present study was that paeoniflorin was able to inhibit proliferation and promote apoptosis of MM cells via inhibition of MMP-2 and upregulation of miR-29b. Understanding the precise role of paeoniflorin may advance knowledge regarding MM, and may be beneficial for future treatment.

\section{References}

1. Fujisawa M, Seike K, Fukumoto K, Suehara Y, Fukaya M, Sugihara H, Takeuchi M and Matsue K: Oligoclonal bands in patients with multiple myeloma: Its emergence per se could not be translated to improved survival. Cancer Sci 105: 1442-1446, 2014

2. Ma Y, Li Z, Wang Y and Feng J: Brucine induces the apoptosis of U266 multiple myeloma cells by phosphorylation of c-Jun. Mol Med Rep 7: 481-484, 2013.

3. Qiao M, Wu D, Carey M, Zhou X and Zhang L: Multi-scale agent-based multiple myeloma cancer modeling and the related study of the balance between osteoclasts and osteoblasts. PLoS One 10: e0143206, 2015.

4. He X, Yang K, Chen P, Liu B, Zhang Y, Wang F, Guo Z, Liu X, Lou J and Chen H: Arsenic trioxide-based therapy in relapsed/refractory multiple myeloma patients: A meta-analysis and systematic review. Onco Targets Ther 7: 1593-1599, 2014.

5. Zhang J, Ai L, Lv T, Jiang X and Liu F: Asiatic acid, a triterpene, inhibits cell proliferation through regulating the expression of focal adhesion kinase in multiple myeloma cells. Oncol Lett 6: 1762-1766, 2013.

6. Garzon R, Calin GA and Croce CM: MicroRNAs in cancer. Annu Rev Med 60: 167-179, 2009.

7. Cao L, Wang $\mathrm{H}$ and Wang F: Amyloid- $\beta$-induced matrix metalloproteinase-9 secretion is associated with retinal pigment epithelial barrier disruption. Int J Mol Med 31: 1105-1112, 2013.

8. Vacca A, Ribatti D, Presta M, Minischetti M, Ria R, Albini A, Bussolono F and Dammacco F: Bone marrow neovascularization, plasma cell angiogenic potential, and matrix metalloproteinase-2 secretion parallel progression of human multiple myeloma. Blood 93: 3064-3073, 1999.

9. Zdzisinska B, Walter-Croneck A and Kandefer-Szerszen M: Matrix metalloproteinases-1 and -2, and tissue inhibitor of metalloproteinase- 2 production is abnormal in bone marrow stromal cells of multiple myeloma patients. Leuk Res 32: 1763-1769, 2008.

10. Bolkun L, Lemancewicz D, Sobolewski K, Mantur M, Semeniuk J, Kulczynska A, Kloczko J and Dzieciol J: The evaluation of angiogenesis and matrix metalloproteinase-2 secretion in bone marrow of multiple myeloma patients before and after the treatment. Adv Med Sci 58: 118-125, 2013.

11. Chen YP, Jin X, Kong M and Li YM: Pattern of microRNA expression associated with different stages of alcoholic liver disease in rat models. Mol Med Rep 10: 1195-1204, 2014.

12. Vandenboom Ii TG, Li Y, Philip PA and Sarkar FH: MicroRNA and cancer: Tiny molecules with major implications. Curr Genomics 9: 97-109, 2008.

13. Ru P, Steele R, Newhall P, Phillips NJ, Toth K and Ray RB: miRNA-29b suppresses prostate cancer metastasis by regulating epithelial-mesenchymal transition signaling. Mol Cancer Ther 11: 1166-1173, 2012.
14. Zhang YK, Wang H, Leng Y, Li ZL, Yang YF, Xiao FJ, Li QF, Chen XQ and Wang LS: Overexpression of microRNA-29b induces apoptosis of multiple myeloma cells through down regulating Mcl-1. Biochem Biophys Res Commun 414: 233-239, 2011.

15. Hu ZY, Xu L, Yan R, Huang Y, Liu G, Zhou WX and Zhang YX: Advance in studies on effect of paeoniflorin on nervous system. Zhongguo Zhong Yao Za Zhi 38: 297-301, 2013 (In Chinese).

16. Fang S, Zhu W, Zhang Y, Shu Y and Liu P: Paeoniflorin modulates multidrug resistance of a human gastric cancer cell line via the inhibition of NF-אB activation. Mol Med Rep 5: 351-356, 2012.

17. Zhang $\mathrm{L}$ and Zhang S: Modulating Bcl-2 family proteins and caspase-3 in induction of apoptosis by paeoniflorin in human cervical cancer cells. Phytother Res 25: 1551-1557, 2011.

18. Livak KJ and Schmittgen TD: Analysis of relative gene expression data using real-time quantitative PCR and the 2(-Delta Delta C(T)) method. Methods 25: 402-408, 2001.

19. Chen YF, Wu KJ and Wood WG: Paeonia lactiflora extract attenuating cerebral ischemia and arterial intimal hyperplasia is mediated by paeoniflorin via modulation of VSMC migration and Ras/MEK/ERK signaling pathway. Evid Based Complement Alternat Med 2013: 482428, 2013.

20. Wang XS, Shi Q, Williams LA, Shah ND, Mendoza TR, Cohen EN, Reuben JM, Cleeland CS and Orlowski RZ: Longitudinal analysis of patient-reported symptoms post-autologous stem cell transplant and their relationship to inflammation in patients with multiple myeloma. Leuk Lymphoma 56: 1335-1341, 2015.

21. Trepel M, Martens V, Doll C, Rahlff J, Gösch B, Loges S and Binder M: Phenotypic detection of clonotypic B cells in multiple myeloma by specific immunoglobulin ligands reveals their rarity in multiple myeloma. PLoS One 7: e31998, 2012.

22. Hu S, Sun W, Wei W, Wang D, Jin J, Wu J, Chen J, Wu H and Wang Q: Involvement of the prostaglandin E receptor EP2 in paeoniflorin-induced human hepatoma cell apoptosis. Anticancer Drugs 24: 140-149, 2013.

23. Hung JY, Yang CJ, Tsai YM, Huang HW and Huang MS: Antiproliferative activity of paeoniflorin is through cell cycle arrest and the Fas/Fas ligand-mediated apoptotic pathway in human non-small cell lung cancer A549 cells. Clin Exp Pharmacol Physiol 35: 141-147, 2008.

24. Yang JL, Lin JH, Weng SW, Chen JC, Yang JS, Amagaya S, Funayana S, Wood WG, Kuo CL and Chung JG: Crude extract of Euphorbia formosana inhibits the migration and invasion of DU145 human prostate cancer cells: The role of matrix metalloproteinase-2/9 inhibition via the MAPK signaling pathway. Mol Med Rep 7: 1403-1408, 2013.

25. Han X, Jin D, Zheng G, Luo Y and Cai Z: Astrocytoma development following complete multiple myeloma remission in a 49-year-old patient: A case report. Exp Ther Med 6: 509-512, 2013.

26. Harford-Wright E, Lewis KM, Ghabriel MN and Vink R: Treatment with the NK1 antagonist emend reduces blood brain barrier dysfunction and edema formation in an experimental model of brain tumors. PLoS One 9: e97002, 2014.

27. Yang JH, Wu MY, Chen MJ, Chen SU, Yang YS and Ho HN: Increased matrix metalloproteinase-2 and tissue inhibitor of metalloproteinase-1 secretion but unaffected invasiveness of endometrial stromal cells in adenomyosis. Fertil Steril 91 (5 Suppl): 2193-2198, 2009.

28. Zhang L, Dong Y, Sun Y, Chen T and Xu Q: Role of four major components in the effect of Si-Ni-San, a traditional Chinese prescription, against contact sensitivity in mice. J Pharm Pharmacol 58: 1257-1264, 2006.

29. Kagiya T and Nakamura S: Expression profiling of microRNAs in RAW264.7 cells treated with a combination of tumor necrosis factor alpha and RANKL during osteoclast differentiation. J Periodontal Res 48: 373-385, 2013.

30. Sugio A, Iwasaki M, Habata S, Mariya T, Suzuki M, Osogami H, Tamate M, Tanaka R and Saito T: BAG3 upregulates Mcl-1 through downregulation of miR-29b to induce anticancer drug resistance in ovarian cancer. Gynecol Oncol 134: 615-623, 2014.

31. Rossi M, Pitari MR, Amodio N, Di Martino MT, Conforti F, Leone E, Botta C, Paolino FM, Del Giudice T, Iuliano E, et al: miR-29b negatively regulates human osteoclastic cell differentiation and function: Implications for the treatment of multiple myeloma-related bone disease. J Cell Physiol 228: 1506-1515, 2013. 